\title{
APPARENT CLUSTERS OF UNEXPLAINED INFANT DEATHS
}

F ive unexplained infant deaths, attributed to Sudden Infant Death Syndrome, occurred in the Coffs Harbour local government area [LGA] between April and July 1990. Subsequently, 18 apparent SIDS deaths between January and October 1990 were noted in the Blacktown LGA. The deaths were investigated by the Epidemiology Branch and the North Coast Region and Western Sector Public Health Units.

Sudden Infant Death Syndrome (SIDS), is defined as the sudden death of an infant or young child which is unexpected by history and in which a thorough postmortem examination fails to demonstrate an adequate cause of death. The cause of SIDS is unknown, but it appears to be associated with low birthweight, young maternal age, maternal smoking, and low socioeconomic status. The peak incidence is in the second and third months of life, with 80-90 per cent of the total incidence in the first six months of life $e^{1}$. SIDS is more common in winter and may occur in clusters 2 . It is more common in males. Recent research has focused on possible relationships with ambient temperature $e^{4,5}$ and with sleeping position, several studies having suggested SIDS is associated with sleeping in the prone position ${ }^{6 \cdot 9}$.

SIDS is a major cause of infant death in NSW. Excluding congenital anomalies and perinatal conditions, it accounts for about 70 per cent of infant deaths. From 1981 to 1988 , the number of deaths attributed to SIDS ranged from 141 (in 1981) to 205 (in 1986). The lowest and highest levels also occurred in these years - the rates ranging from 1.7 to 2.4 per 1000 live births. In general, SIDS was more frequent in winter (June, July and August).

A major difficulty in the investigation of SIDS is the diagnosis. It is strictly a diagnosis of exclusion which often is not clearcut, especially where some pathology is detected at autopsy, and where it is difficult to assess the extent to which this pathology can explain the death. Even potentially lethal conditions may be difficult to detect at infant autopsies.

Although unexplained deaths resembling SIDS were recognised as long ago as $1834^{10}$, Sudden Infant Death Syndrome was first proposed as a diagnostic term in $1969^{11}$, and came into general use only in the late $1970 \mathrm{~s}$ with the introduction of the 9 th Revision of the International Classification of Diseases. Since that time SIDS has tended to displace some other diagnoses that may have been ascribed to unexplained infant deaths.

\section{INVESTIGATION OF UNEXPLAINED INFANT DEATHS}

Unexpected infant deaths in NSW are reported to the coroner. A police officer investigates the death scene and compiles a report which may include accounts of interviews with the parents and other witnesses. The coroner invariably orders an autopsy. In the Sydney metropolitan area autopsies are done at the Institute of Forensic Medicine (at the Coroner's Court in Glebe) or the Department of Forensic Pathology, Institute of Clinical Pathology and Medical Research (at Westmead Hospital). Elsewhere in the State the autopsies are done by Government Medical Officers. In most districts the
GMO is a general practitioner with basic training in autopsy techniques, but in some areas forensic autopsies are done by local pathologists. The histopathology from country cases is referred to the Institute of Forensic Medicine for reporting and toxicology is done by the Division of Analytical Laboratories.

The Blacktown and Coffs Harbour investigations set out to answer the following questions: (i) Was the occurrence of SIDS in excess of expectation? (ii) What were the characteristics of the cases, and did they have any common features that might be amenable to prevention?

For both investigations 1981-87 NSW mortality data were used to establish the incidence of SIDS (both Statewide and in the Coffs Harbour and Blacktown LGAs) and to estimate the expected frequency. Efforts were made to ascertain all infant deaths attributed to SIDS during the index periods, and the observed numbers of deaths were compared with the expectations. Individual cases were reviewed in detail, including an inspection of coroners' records and extraction of data from medical records.

The Coffs Harbour investigations included interviews with the infants' parents and a review of environmental factors, particularly the use of agricultural chemicals on banana plantations and their levels in potable water. Respiratory disease admissions and infant attendances at the Coffs Harbour District Hospital's accident and emergency unit for apnoea, gastro-oesophageal reflux or "acute life-threatening events" (also known as "nearmiss SIDS") also were reviewed.

\section{FINDINGS - COFFS HARBOUR}

A total of 10 deaths of Coffs Harbour LGA residents were attributed to SIDS from 1981 to 1987 . No SIDS deaths were recorded in the years 1981-83, and four were recorded in 1986, paralleling the high Statewide level in that year. The annual incidence of SIDS in the Coffs Harbour LGA ranged from zero to 6.0 cases per 1000 live births. One SIDS death was recorded in 1988 and none in 1989.

Based on the 1987 NSW incidence of 2.0 per 1000 live births, 1.3 cases of SIDS are expected to occur in the Coffs Harbour LGA over a calendar year. Five deaths were attributed to SIDS between April and July 1990. Compared with the 1987 Statewide incidence, the probability of five or more cases occurring by chance in the Coffs Harbour LGA over a calendar year was less than 1 per cent.

The diagnosis of SIDS was substantiated in three of the five cases and SIDS was considered to be the most likely cause of death in one of the other two cases. In the remaining case there was autopsy evidence of pulmonary infection. The deaths occurred at widely dispersed sites and the histories of the individual cases did not suggest any common or preventable factors.

There was no toxicological evidence that any of the agricultural chemicals - which comprised fungicides, organophosphorous compounds, paraquat and an organic fertiliser - could cause SIDS, and it was 
impossible to suggest a plausible biological mechanism to implicate these substances. While the Coffs Harbour Shire Council regularly sampled the reticulated water supply for testing by the Health Department's Pesticide Residue Laboratory in Sydney, the routine testing program included only one of the compounds used on the banana plantations, namely chlorpyrifos. Chlorpyrifos, an organophosphorous compound, had not been detected in the water, but the sampling was not synchronised with pesticide spraying.

The review of respiratory admissions and accident and emergency presentations to the Coffs Harbour District Hospital was largely non-contributory.

\section{FINDINGS - BLACKTOWN}

All unexpected infant deaths in the Blacktown area are referred to the Department of Forensic Pathology at Westmead. The Department's records for January to October 1990 were therefore assumed to include all the relevant deaths from the Blacktown area. Eighteen deaths were attributed to SIDS during this period, representing an incidence 1.57 times that of the remainder of the State from 1983 to 1987 . Compared with the 1983-87 Statewide incidence, the probability of 18 or more cases occurring by chance in the Blacktown LGA over a calendar year was less than 1 per cent.

Data are incomplete from 1988 onwards. Provisional data from the Department of Forensir. Pathology show that the 1988-90 incidence of deaths attributed to SIDS in the Blacktown LGA ranged from 4.6 to 5.8 deaths per 1000 live births. If the SIDS incidence in NSW remained constant at the 1988 rate of 2.2 per 1000 live births over this period, the incidence of SIDS in the Blacktown LGA would be at least double that of the State as a whole.

The 18 cases comprised 11 male and seven female infants of whom seventeen were less than six months old. Nine had had an upper respiratory tract infection in the week before death. Thirteen cases occurred in the cooler months (April to August). Half the mothers were aged 21 years or less, and 16 had had at least one previous pregnancy.

Again, there were no features to suggest a common and preventable cause. Seven of the infants had evidence of respiratory tract infection at autopsy, although two of these had no documented respiratory symptoms before death. A respiratory virus was isolated at autopsy from three of the seven. There were no remarkable features to account for the deaths or to link the cases.

\section{LESSONS AND SUBSEQUENT ACTION}

Two lessons emerged from the investigations. the first was the lack of any mechanism for obtaining timely surveillance data on the occurrence of unexpected infant deaths. While the apparent clusters in the Coffs Harbour and Blacktown LGAs were brought to the attention of the Epidemiology Branch and the local Public Health Units, contemporary information on the Statewide occurrence of such deaths was unavailable, and therefore the apparent clusters could be evaluated only in the context of previous years' data on SIDS. The second lesson was the variation in autopsy procedures, with the resultant likelihood of variation in the use of SIDS as a diagnostic label.
Two major initiatives have followed from the investigations. First, the (then) Minister for Health, Mr Peter Collins, announced that a Statewide surveillance program of unexpected infant deaths would be set up by the Health Department. The NSW Sudden Infant Death Syndrome Advisory Committee, chaired by the State Coroner, $\mathrm{Mr}$ Kevin Waller, has been re-convened with a primary objective of advising on and facilitating the establishment of the surveillance program. Central to the program is the provision of an expert autopsy service for all infant deaths reported to the coroner, irrespective of where they occur.

Second, in conjunction with other agencies, the North Coast Region Public Health Unit has undertaken a review of agricultural chemical use and monitoring in the Region, and the Coffs Harbour Shire Council's reticulated water-testing program has been synchronised with the pesticide spraying cycle.

There have been further developments nationally. In April 1991 a workshop jointly sponsored by the National SIDS Council, the Royal College of Pathologists of Australia and the Victorian Forensic Pathology Institute led to the design of a national SIDS autopsy protocol, with a first draft due to be presented in October. In May, Tasmanian data supporting Dutch, British and New Zealand findings on the relationship between SIDS and the prone sleeping position was published.

Mass-media programs in New Zealand, which has the highest known SIDS incidence in the world, have directed attention to sleeping position, breastfeeding and maternal non-smoking; contemporaneous with these programs the SIDS incidence has dropped appreciably, suggesting a possible preventive effect. Following from these developments, the Menzies Foundation convened a meeting of experts in July to decide on policy recommendations about infants' sleeping positions.

Peter Lewis, Public Health Officer

Michael Frommer, Deputy Director Christine Roberts, Public Health Officer

Pamela Adelson, Epidemiology Officer

Epidemiology \& Health Services Evaluation Branch NSW Health Department

Margrette Young, North Coast Region Public Health Unit

1. Peterson DR. Sudden infant death syndrome, IN Bracken MB (ed), Perinatal Epidemiology. Oxford University Press, Oxford, 1984

2. Grimson RC et al. Searching for hierarchical clusters of disease: spatial patterns of sudden infant death syndrome. Social Science and Medicine, 1981; 15D: $287-93$.

3. Mellins RB and Haddad GG. Sudden infant death syndrome, IN Behrman RE, Vaughan VC and Nelson WE (eds), Nelson Textbook of Paediatrics, 13th RE, Vaughan VC and Nelson WE (eds), Nelson Textbo

4. Murphy MFG and Campbell MJ. Sudden infant death syndrome and 4. Murphy MFG and Campbell MJ. Sudden infant death syndrome and
environmental temperature: an analysis using vital statistics. $J$ of Epidem and Comm Health, 1987; 41:63-71.

5. Campbell MJ. Sudden infant death syndrome and environmental temperature: further evidence for a time-lagged relationship. Med. J of Aust, 1989; 151: 365-7.

6. de Jonge GA et al. Cot death and prone position in The Netherlands. Brit Med J, 1989; 298: 722.

7. Fleming $\mathrm{PJ}$ et al. Interaction between bedding and sleeping position in the sudden infant death syndrome: a population-based case-control study. Brit Med. J. 1990; 301: 85-9.

8. Mitchell EA et al. Results from the first year of the New Zealand cot death study. NZ Med J, 1991; 104: 71-6.

9. Dwyer T et al. Prospective cohort study of prone sleeping position and

9. Dwyer Tet al. Prospective cohort study of prone sleepin

10. Fearn SW. Sudden unexplained death of children (letter).

10. Fearn SW. Sudde

Lancet, 1934 ; i: 246

11. Emery $\int$. Is sudden infant death syndrome a diagnosis? Or is it just a diagnostic dustbin? Brit Med J, 1989; 299: 1240. 